\title{
An incidental finding in a 34-year-old male under investigation for haemoptysis
}

\author{
D. Pinot*,+, D. Breen ${ }^{\#,+}$, J-M. Peloni*, J-Y. Gaubert ${ }^{\dagger}$, H. Dutau* ${ }^{\#}$ and D. Vervloet*
}

\section{CASE REPORT}

A 34-yr-old male presented with a 1-week history of a productive cough associated with small volume haemoptysis. This was preceded by a 3-month history of a chronic nonproductive cough and $6 \mathrm{~kg}$ weight loss. He denied chest pain, fever or night sweats. He admitted to increasing fatigue, but denied arthalgia, myalgia or skin rash. His past medical history was noncontributory.

The patient worked in the construction industry and smoked 20 cigarettes per day. He admitted to illicit drug usage, both cannabis and cocaine on a regular basis.

On examination, he was afebrile and haemodynamically stable. There was evidence of recent weight loss. His respiratory rate was 18 breaths $\cdot \mathrm{min}^{-1}$ and oxygen saturations were $99 \%$ in room air. Lung examination was unremarkable.

Laboratory examination included the following: white blood cell count $8.28 \mathrm{~g} \cdot \mathrm{L}^{-1}$ with $43 \%$ neutrophils; haemoglobulin $16.3 \mathrm{~g} \cdot \mathrm{L}^{-1}$; haematocrit $47 \%$; erythrocyte sedimentation rate $1 \mathrm{~mm}$ in the first $\mathrm{h}$ and $\mathrm{C}$-reactive protein $1.55 \mathrm{mg} \cdot \mathrm{L}^{-1}$. The results of renal function tests and biochemistry were within normal limits. Sputum specimens for culture and sensitivity and total blood count were negative. A plain chest radiograph and computer tomography (CT) of the thorax were performed (fig. 1a-c). Further investigations including $\alpha_{1}$ antitrypsin, pulmonary function and bronchoprovocation tests were normal. Oral examination and gastroscopy were within normal limits and did not reveal any source of bleeding. A bronchoscopy was performed (fig. 1d) and aspirates demonstrated a positive culture of Haemophilus influenzae, which was sensitive to amoxicillin.
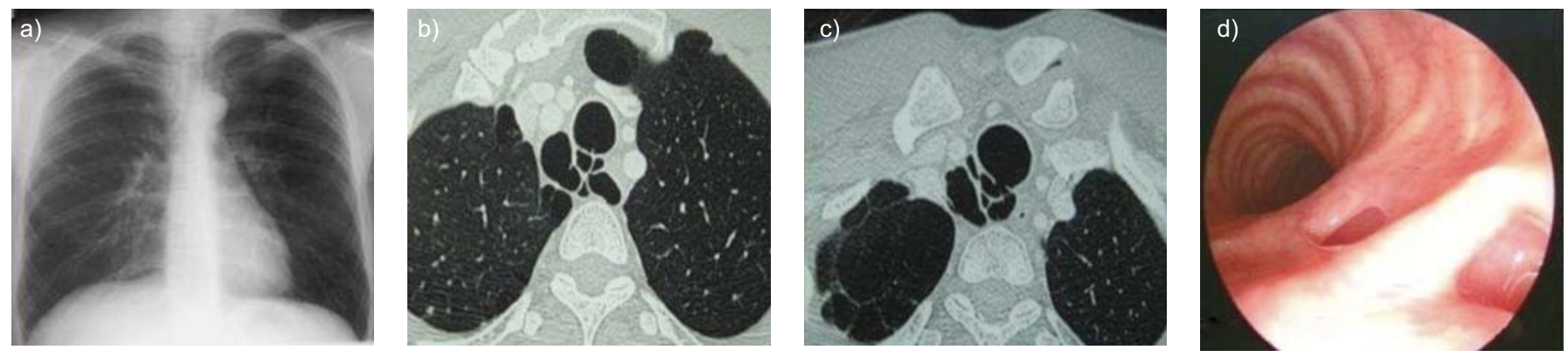

FIGURE 1. a) A chest radiograph, b and c) computed tomography images at the level of the trachea and d) endoscopic view of the trachea at the time of bronchoscopy

\section{BEFORE TURNING THE PAGE, INTERPRET THE RADIOGRAPH, COMPUTED TOMOGRAPHY SCAN AND ENDOSCOPE IMAGES, AND SUGGEST A DIAGNOSIS.}

\footnotetext{
${ }^{*}$ Respiratory Dept, ${ }^{\#}$ Thoracic Endoscopy Unit, and "Dept of Radiology, Sainte Marguerite University Hospital, Marseille, France. ${ }^{+}$These authors contributed equally to the present study. STATEMENT OF INTEREST: None declared. 


\section{Diagnosis: The radiological and endoscopic images demonstrate a complex defect along the posterior tracheal wall consistent with acquired tracheal diverticulum.}

Tracheal diverticulum is a rare benign condition characterised by invaginations of the tracheal wall resulting in paratracheal air cysts [1]. It may be considered as part of a spectrum of conditions, which include pharyngocele, laryngocoele, apical herniation and bullae, and Zenker's diverticulum [2]. These may all share a similar pathological mechanism. It is infrequently reported in clinical practice with only 30 reports in the literature to date. The reported incidence of this condition varies from series to series and depends, in part, on the diagnostic tool used. The reported incidence was $0.3 \%$ and $2 \%$ in two series, which diagnosed the diverticula by bronchoscopy and CT, respectively $[3,4]$. In an autopsy series, the incidence was reported at $1 \%$ [5].

Tracheal diverticulum can be divided into congenital and acquired forms [6, 7]. Congenital diverticulum are more common in males than females and are usually single. The mechanism remains unclear, but it likely develops secondary to altered embryonic development where a malformed supernumerary branch of the trachea developed. Thus, it is therefore composed of all the layers of the tracheal wall [7] and are usually located $4-5 \mathrm{~cm}$ from the vocal cords on the right lateral wall of the trachea. They are frequently filled with mucus, which may become superinfected as the communication with the trachea is often narrow and results in poor drainage of the secretions. Tracheal diverticulum are occasionally associated with other congenital abnormalities [7, 8].

Acquired tracheal diverticulum have an equal sex ratio and may occur anywhere in the trachea, but have a tendency to develop at the junction between the extrathoracic and intrathoracic trachea on the posterolateral wall where it is at its weakest. The mechanism of development is related to raised intrathoracic pressure, which causes the mucosa to herniate at this level. Therefore, they consist of respiratory epithelium without smooth muscle or cartilage and may be single or multiple. Unlike congenital lesions, the mouth of an acquired diverticulum is wide and hence patients are often asymptomatic and infection free.

Clinically, the majority of patients with congenital or acquired diverticula are asymptomatic [9]. However, as mentioned previously, the diverticulum can retain secretions and become secondarily infected resulting in recurrent episodes of tracheobronchitis [10]. This may be associated with cough, dyspnoea and haemoptysis. In the current case, the patient presented with cough and haemoptysis, but the present authors believe that this was more likely related to regular illicit drug use and not directly associated with the diverticulum as there was no evidence of bleeding at the level of the tracheal defect at the time of bronchoscopy. Recently, there is a greater awareness of the detrimental effects to regular smokers of illicit drugs, including cannabis and cocaine, on the respiratory system [11-13]. Indeed, cough may play two roles in diverticulum, either an aetiological factor in their development or a symptom secondary to the lesion. In the present case, it is possible that the patient had
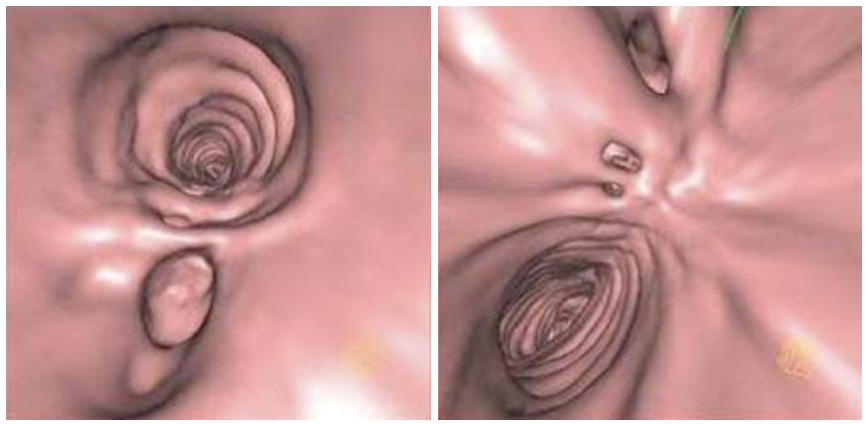

FIGURE 2. Virtual bronchoscopy images of the posterior membranous tracheal wall.
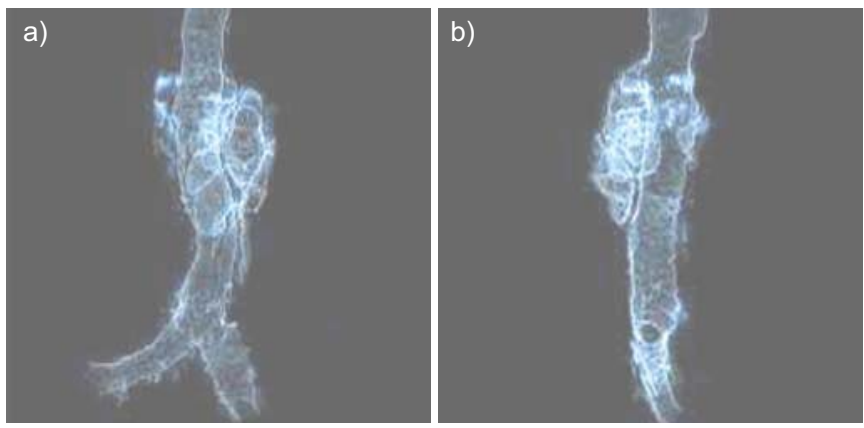

FIGURE 3. Computed tomography reconstruction of the a) frontal and b) sagittal view of the posterior tracheal wall. A volume rendering console was used (Leonardo Siemens, Munich, Germany)
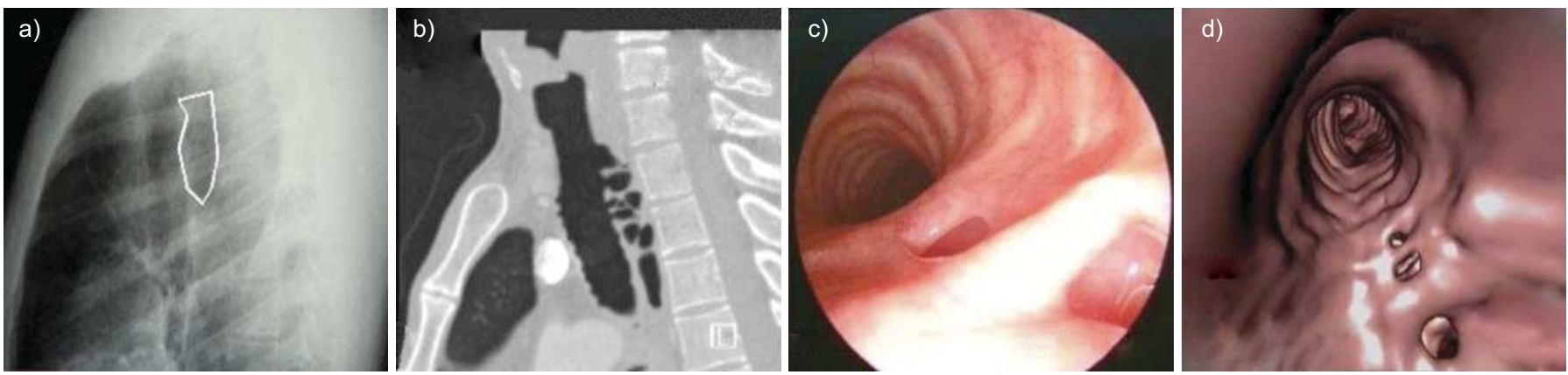

FIGURE 4. a) A lateral chest radiograph with the paraseptal air space marked. b) Computed tomography (CT) demonstrating multiple complex lesions, some of which are in communication with the trachea. c) Endoscopic view of the trachea at the time of bronchoscopy and d) spiral-CT virtual bronchoscopy reconstruction of the trachea. 
recurrent episodes of bronchitis due to his habitual drug usage resulting in raised intrathoracic pressures. Rarely, a large diverticulum can compress the recurrent laryngeal nerve and result in dysphonia [5]. Finally, there are case reports of respiratory distress due to selective intubation of the diverticulum and perforation of the lesion during airway manipulation resulting in a pneumomediastinum [14].

A physician should maintain a high index of suspicion for diverticulum in patients with respiratory symptoms, particularly if the plain film radiography and spirometry are normal. Although partracheal collections of air can occasionally be seen on chest radiography, further investigations are required to assess the origin and size of the lesion. This includes barium studies to identify if the paratracheal air cyst is associated with the oesophagus, for example a Zenker's diverticulum. Further investigations include traditional bronchoscopy and CT. In some cases, bronchoscopy may fail to diagnose the diverticulum, particularly if the mouth of the lesion is small, such as in congenital cases [2]. An additional imaging tool which may be utilised if the diagnosis remains elusive or to define the extent of the diverticulum is three-dimensional reconstruction CT to create a virtual bronchoscopy (figs 2-4) [9].

The differential diagnosis includes pharyngocele, laryngocoele, Mounier-Kuhn syndrome, apical herniation and bullae, emphysema and Zenker's diverticulum, all of which can be distinguished from each other based on previous investigations [2].

Treatment options can be divided into medical and surgical [1]. The majority of patients are asymptomatic and require no specific intervention. In symptomatic cases, physiotherapy to assist drainage of the diverticulum and prevent infections may be beneficial. If infections are problematic, antibiotics and mucolytics can be administered. Surgery should be reserved for young or symptomatic patients. Surgical options include laser cauterisation via rigid bronchoscopy and open repair via a lateral cervical approach.

In conclusion, a case of an acquired tracheal diverticulum in a 34 -yr-old male with habitual drug usage was reported. The present authors believe that his symptoms were not related to the diverticulum, but it is possible that the chronic cough or bronchitis related to habitual drug use may have been an aetiological factor in its development. The patient was treated with antibiotics and counselled for his illicit drug usage. He had no further episodes of haemoptysis while in hospital and was lost to follow-up.

Although rarely reported in clinical practice or the literature, the incidence of tracheal diverticulum may be as high as $2 \%$. Therefore, the current authors believe that clinicians should include tracheal diverticulum in their differential diagnosis of patients presenting with respiratory symptoms, particularly if the initial chest radiograph and spirometry are normal.

\section{REFERENCES}

1 Soto-Hurtado EJ, Peñuela-Ruíz L, Rivera-Sánchez I, TorresJiménez J. Tracheal diverticulum: a review of the literature. Lung 2006; 184: 303-307.

2 Djamouri F, Le Pimpec Barthes F, Pheulpin G, Grignet JP, Carnot F, Riquet M. Kystes aériens d'origine trachéale: problèmes nosologiques et fréquence réelle. [Air-filled cysts of tracheal origin: nosological problems and actual frequency.]. Rev Mal Respir 2002; 19: 523-526.

3 MacKinnon D. Tracheal diverticula. J Pathol Bacteriol 1953; 65: 513-517.

4 Goo JM, Im JG, Ahn JM, et al. Right paratracheal air cysts in the thoracic inlet: clinical and radiologic significance. AJR Am J Roentgenol 1999; 173: 65-70.

5 Caversaccio MD, Becker M, Zbaren P. Tracheal diverticulum presenting with recurrent laryngeal nerve paralysis. Ann Otol Rhinol Laryngol 1998; 107: 362-364.

6 Hernandez Pérez JM, Pérez Negrin L, Batista Martin JJ, Gonzalvo Hernandez F. Intratracheal diverticulum. J Bronchol 2005; 12: 104-105.

7 Restrepo S, Villamil MA, Rojas IC, et al. Association of two respiratory congenital anomalies: tracheal diverticulum and cystic adenomatoid malformation of the lung. Pediatr Radiol 2004; 34: 263-266.

8 Morel V, Corbineau H, Lecoz A, et al. Two cases of "asthma" revealing a diverticulum of Kommerell. Respiration 2002; 69: 456-460.

9 Polverosi R, Carloni A, Poletti V. Tracheal and main bronchial diverticula: the role of CT. Radiol Med 2008; 113: 181-189.

10 Early EK, Bothwell MR. Congenital tracheal diverticulum. Otolaryngol Head Neck Surg 2002; 127: 119-121.

11 Aldington S, Williams $M$, Nowitz $M$, et al. Effects of cannabis on pulmonary structure, function and symptoms. Thorax 2007; 62: 1058-1063.

12 Taylor DR, Hall W, Thoracic Society of Australia and New Zealand, Respiratory health effects of cannabis: position statement of the Thoracic Society of Australia and New Zealand. Intern Med J 2003; 33: 310-313.

13 Tashkin DP, Khalsa ME, Gorelick D, et al. Pulmonary status of habitual cocaine smokers. Am Rev Respir Dis 1992; 145: 92-100.

14 Moller GM, ten Berge EJ, Stassen CM. Tracheocele: a rare cause of difficult endotracheal intubation and subsequent pneumomediastinum. Eur Resp J 1994; 7: 1376-1377. 